\title{
Current Insights Into the Pathophysiology of Multisystem Inflammatory Syndrome in Children
}

\author{
Laura A. Vella ${ }^{1,2} \cdot$ Anne H. Rowley ${ }^{3,4}$ \\ Accepted: 29 September 2021 / Published online: 19 October 2021 \\ (c) The Author(s), under exclusive licence to Springer Science+Business Media, LLC, part of Springer Nature 2021
}

\begin{abstract}
Purpose of Review We highlight the new clinical entity multisystem inflammatory syndrome in children (MIS-C), the progress in understanding its immunopathogenesis, and compare and contrast the clinical and immunologic features of MIS-C with Kawasaki disease (KD).

Recent Findings Studies show immune dysregulation in MIS-C including T lymphocyte depletion and activation, $\mathrm{T}$ cell receptor Vbeta skewing, elevated plasmablast frequencies, increased markers of vascular pathology, and decreased numbers and functional profiles of antigen-presenting cells.

Summary MIS-C is a late manifestation of infection with SARS-CoV-2 associated with marked immune activation and many potential mechanisms of immunopathogenesis. MIS-C and KD have clinical similarities but are distinct. Myocardial dysfunction with or without mild coronary artery dilation can occur in MIS-C but generally corrects within weeks. In contrast, the coronary arteries are the primary target in $\mathrm{KD}$, and coronary artery sequelae can be lifelong. Supportive care and anti-inflammatory therapy appear to hasten improvement in children with MIS-C, and there is hope that vaccines will prevent its development.
\end{abstract}

Keywords MIS-C $\cdot$ Kawasaki disease $\cdot$ Pediatric $\cdot$ SARS-CoV-2 $\cdot$ Inflammatory syndrome

\section{Introduction}

SARS-CoV-2 infection has provided many unexpected events for the medical profession, not the least of which has been the pediatric experience. Unlike other respiratory viral pathogens such as RSV or influenza, this new human virus does not commonly cause severe disease in young children $1 \bullet$. Moreover, despite children rarely requiring

Anne H. Rowley

a-rowley@northwestern.edu

1 Division of Infectious Diseases, Department of Pediatrics, Children's Hospital of Philadelphia, University of Pennsylvania Perelman School of Medicine, Philadelphia, PA 19104, USA

2 Institute for Immunology, University of Pennsylvania Perelman School of Medicine, Philadelphia, PA 19104, USA

3 Division of Infectious Diseases, Department of Pediatrics, The Ann \& Robert H. Lurie Children's Hospital of Chicago, 225 E Chicago Avenue, Box 20, Chicago, IL 60611, USA

4 Northwestern Feinberg School of Medicine, Chicago, IL 60611, USA hospitalization for acute COVID-19, it became clear over time that a small subset of children developed a serious illness at a median of 4 weeks after the acute infection, manifested by prolonged fever, hypotension, myocardial dysfunction, and marked increase in inflammatory markers in the blood. Initially termed pediatric multisystem inflammatory syndrome (PIMS) in Europe [2•], this clinical illness became known as multisystem inflammatory syndrome in children (MIS-C) in the USA [3•]. In this review, we focus on what is presently known about the epidemiology, immunopathogenesis, and treatment of this unique illness. We also describe the differences between MIS-C and Kawasaki disease (KD), a pediatric illness with some clinical similarities to MIS-C.

\section{Epidemiology}

Identifying the precise epidemiology of MIS-C is hampered by the lack of a specific case definition. Current case definitions are very broad; children who recently or previously were infected with SARS-CoV-2 and develop a multisystem illness due to infections or inflammatory conditions 
unrelated to SARS-CoV-2 or due to acute COVID-19 can fulfill current case definitions, resulting in overdiagnosis [3•, $4,5 \bullet]$. However, certain epidemiologic features are consistently reported. The median age of children with MIS-C is 9 years $[2 \bullet, 3 \bullet, 4,6]$. MIS-C occurs a median of 27 days (IQR 21-36 days) after preceding SARS-CoV-2 infection [7••], and an increase in cases has occurred about 1 month (range 2-5 weeks) following peaks of COVID-19 in individual geographic areas $[\mathbf{7} \bullet$ ]. If RT-PCR for SARS-CoV-2 from the respiratory tract is positive in children with MIS$\mathrm{C}$, the cycle threshold $(\mathrm{Ct})$ values are high, suggesting low viral load at that site at the time of clinical presentation $[7 \bullet \bullet, 8 \bullet \bullet$. About $60 \%$ of affected children in the USA have been reported to be Hispanic or non-Hispanic Black, with

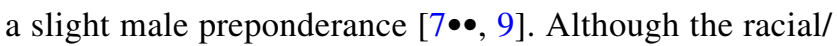
ethnic distribution most likely reflects COVID-19-related health disparities, a genetic predisposition to MIS-C has not been excluded [9].

Adults can develop an illness similar to MIS-C following COVID-19 infection, called multisystem inflammatory syndrome in adults, or MIS-A; it may be underdiagnosed $[10,11]$.

\section{Immunology}

MIS-C is associated with high levels of inflammation and responds to anti-inflammatory therapies; it is therefore presumed to be immune-mediated. The immunopathogenesis of MIS-C remains unknown, but substantial progress has been made in defining the features of immune dysregulation in MIS-C. Once the syndrome was recognized, global research efforts collected blood samples from patients with MIS-C to assess plasma cytokines, changes in innate and adaptive immune cells, and profiles of antibodies against

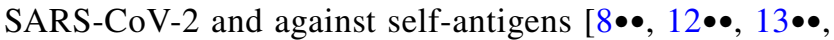
$14 \bullet \bullet, 15 \bullet, 16 \bullet, 17 \bullet, 18 \bullet, 19 \bullet]$. Studies were undertaken in pandemic conditions and in the setting of an evolving field, and therefore, each had limitations. These included lack of ideal comparator groups (acute COVID-19 subjects or pediatric healthy controls), comparator samples that were not contemporaneously collected, sample collection after start of immunosuppressive therapies, low numbers of cell events captured, and small sample sizes. Moreover, some studies include a substantial percentage of patients diagnosed with MIS-C who were negative for SARS-CoV2-specific antibodies, suggesting they may either have had acute COVID-19 if they had a positive respiratory PCR assay, or had an alternative diagnosis. Still, several themes emerged (Table 1).

Changes in Plasma Cytokine and Protein Profiles To identify both diagnostic tools and therapeutic targets in MIS-C, multiple groups assessed the profiles of plasma cytokines and other plasma proteins $[12 \bullet \bullet, 13 \bullet \bullet, 14 \bullet \bullet, 16 \bullet, 17 \bullet, 18 \bullet, 19 \bullet$, $20 \bullet, 21-23]$. Consistent with multisystem inflammation, patients with MIS-C variably demonstrated elevations above the normal range in multiple plasma cytokines, including IL-6, IL-8, IFN $\gamma$, IL-17, TNFa, IFN $\gamma$, and IL-10 [13••, $14 \bullet \bullet, 16 \bullet, 17 \bullet, 18 \bullet, 21,24]$, and several groups have identified changes in interferon responsiveness pathways [20•, 25, 26]. Cytokine levels did not clearly sort with patients who remained PCR positive at presentation versus those who were PCR negative [19•], and although some cytokines such as IFN $\gamma$, IL-10, and TNFa are more prominent in MIS-C and therefore serve as indicators of possible immunopathogenesis, there remains overlap with the cytokine profiles of

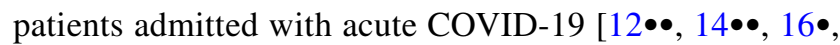
$17 \bullet, 18 \bullet]$.

Broader plasma protein measurements have identified candidate markers for MIS-C, serving as potential clues into aspects of disease pathogenesis. A proteomic study of MIS-C found that phospholipase A2 (PLA2G2A) - an enzyme component of many inflammatory pathways was the plasma protein most elevated in MIS-C compared to healthy controls and typically higher in MIS-C than in acute pediatric COVID-19 [20•]. Increased PLA2G2A was observed in smaller MIS-C cohorts by two other groups $[15 \bullet, 23]$ and has been shown to correlate with thrombocytopenia and with elevations in a complement biomarker of the tissue microangiopathy observed in MIS-C, soluble C5B9 [17•, 20•]. As such, elevation of PLA2G2A is one of
Table 1 Immunologic findings in MIS-C

\begin{tabular}{ll}
\hline Immune system component & Consistent findings \\
\hline Cytokines & Increases in multiple, including IFN $\gamma$, IL-10, TNFa \\
Proteome & Increased PLA2G2A \\
Monocytes & Decreased HLA-DR \\
Dendritic cells & Decreased pDC \\
B cells & Increased plasmablasts \\
SARS-CoV-2-specific antibody & Normal quantity and neutralization \\
Autoantibody & Detected, but no single autoantibody confirmed across studies \\
T cells & Decreased numbers, increased activation \\
\hline
\end{tabular}


the more consistent research findings in MIS-C. In addition to insights into disease pathogenesis, assessment of plasma proteins has tremendous potential as a diagnostic tool for MIS-C. Studies that pool larger numbers of subjects, compare across similar febrile presentations, and collect plasma before immune modulation will be required to develop such tools further.

Changes in Innate Immune Cells In another consistent finding, children with MIS-C demonstrate decreases in monocyte and dendritic cell subsets, particularly plasmacytoid dendritic cells $[8 \bullet \bullet, 13 \bullet \bullet, 14 \bullet \bullet, 15 \bullet]$. The remaining cells have decreased expression of the class II molecule HLA-

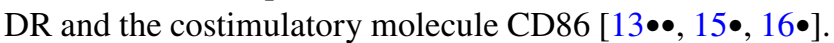
Of note, changes in monocytes and dendritic cells are not exclusive to MIS-C. Reduced plasmacytoid DC have been described in adult and pediatric COVID-19 [14••, 27] and reduced HLA-DR expression on monocytes is well described in critical illness. In sepsis, reduced HLA-DR on monocytes is considered to be a component of "immunoparalysis" because it reduces the capacity for monocytes to present antigen and correlates with worse outcome [28]. To that end, multiple studies have used activating immune modulation such as granulocyte-macrophage colony-stimulating factor (GM-CSF) to increase HLA-DR expression and/or immune responsiveness in sepsis [28, 29]. It is therefore interesting to consider that monocyte and dendritic cell HLA-DR expression in MIS-C appeared to improve instead in the context of therapeutic immunosuppression [13••]. The changes in monocytes and dendritic cells might therefore fit in a broader concept of immune dysregulation - with both aberrantly increased and decreased inflammatory pathways - rather than immune hyporesponsiveness alone.

Changes to Antibodies and B cells in MIS-C The majority of patients diagnosed with MIS-C have been SARS-CoV-2 IgG

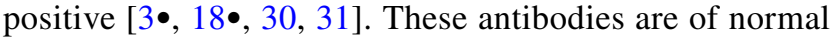
quantity and are able to neutralize virus $[18 \bullet, 31]$. Some quality differences in SARS-CoV-2-specific antibodies have been observed, depending on the comparator group. Patients with MIS-C as compared to children with acute COVID-19 had a greater IgG1:IgG3 ratio of spike-specific antibodies, with enhanced antibody-dependent cellular phagocytosis (ADCP) [18•]. In a separate study, the ADCP ability was on par with convalescent adults [32]. In general, studies of antibody differences between patients with COVID-19 and MIS-C may benefit from convalescent cohorts, as convalescent time periods normalize for the longer time between infection and hospitalization that is a hallmark of MIS-C as compared to acute COVID-19. When compared to convalescent adults, spike-specific antibodies from subjects with severe MIS-C demonstrated an increased ability to activate monocytes [32]. Together, these data suggest differences in the quality of antibody responses to SARS-CoV-2 in MIS$\mathrm{C}$, but whether those differences are due to age, time from infection, or are a reflection of aberrant virus-specific immunity remains unclear.

Profiling of B cell responses in MIS-C has shown increases in circulating plasmablasts $[8 \bullet \bullet, 13 \bullet \bullet, 15 \bullet$, similar to levels typically seen after severe viral infection or live viral vaccinations [33-35]. Of note, the degree of plasmablast elevation is also similar to that observed in acute adult and pediatric COVID-19 [8••], despite the many weeks from initial infection. Further, plasmablast levels are higher than observed in convalescent adults, in whom plasmablast frequencies return to baseline within weeks $[8 \bullet \bullet$. The absence of samples from children in the week(s) before they develop MIS-C limits our ability to know whether these plasmablast responses are persisting from the initial infection or whether they are a newly developed immune response at the time of clinical illness.

The antigenic targets of circulating plasmablasts $\sim 4$ weeks after initial infection have not been identified. A major question is whether these plasmablasts are virus-specific and continuing to expand based on persisting SARS-CoV-2 antigen, reacting nonspecifically to inflammation, or whether they are responding to a non-viral antigen as part of the immunopathogenesis of MIS-C. To understand the potential for autoimmunity as a driver of disease, B cell and antibody responses against self-antigens have been

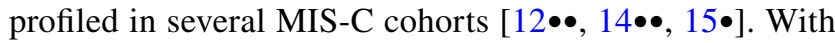
limited numbers, there is some suggestion that antibodies in MIS-C can bind to activated human cardiac microvascular endothelial cells; however, most samples with this finding were obtained after administration of intravenous immunoglobulin [15•]. Autoantibodies that bound self-antigens including endothelial, cardiovascular, and gastrointestinal antigens were also found to be enriched in MIS-C patients as compared to healthy controls, although these studies also included a mixture of pre- and post-IVIg treated subjects [14••]. The largest set of subjects to undergo autoantibody screens included 12 pre-treatment patients with MIS-C compared with the autoantibody profiles of patients with acute SARS-CoV-2, Kawasaki disease, and healthy subjects and found antibodies that bound to proteins involved in immu-

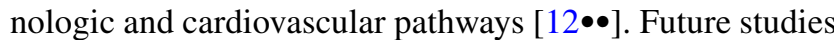
would benefit from increased sample sizes, prioritization of sample collection before administration of IVIg, and inclusion of febrile controls with non-SARS-CoV-2 viral or bacterial illnesses $[36,37]$.

Changes in T cells Clinical laboratory measurements highlighted decreased absolute lymphocyte counts in many patients with MIS-C $[3 \bullet, 8 \bullet \bullet, 38]$. In particular, T cell lymphopenia was observed, with losses in both CD4+ and 


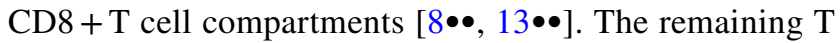
cells demonstrated marked activation, with high-frequency expression of HLA-DR and CD38, particularly in CD8 T cells $[8 \bullet \bullet, 13 \bullet \bullet]$. In addition, deep immune profiling of peripheral blood mononuclear cells directly after isolation demonstrated that the most differentially activated subset was CD8 T cells that express CX3CR1, the fractalkine receptor that enables cells to interact with fractalkine-expressing

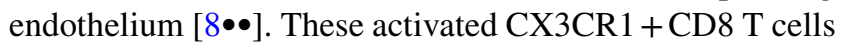
correlated with elevated D-dimer and decreased platelet count and need for vasoactive support [8••]. Finally, several groups have documented that the $\mathrm{T}$ cells remaining in circulation are often enriched for usage of the $\mathrm{T}$ cell receptor beta variable gene 11-2 (TRBV11-2), encoding Vbeta(V $\beta) 21.3$, and enrichment is not present in children with acute COVID$19[15 \bullet, 16 \bullet, 19 \bullet]$. These $\mathrm{V} \beta 21.3+\mathrm{T}$ cells are more likely to be activated and also express CX3CR1 at greater frequencies than T cells without V $\beta 21.3[16 \bullet]$. However, the MHC class that binds the potential superantigen remains incompletely described, and $\mathrm{V} \beta 21.3+\mathrm{T}$ cell activation is seen in both CD4 and CD8 T cells [16•]. In summary, the picture emerging in MIS-C is one of decreased circulating $\mathrm{T}$ cells with those $\mathrm{T}$ cells remaining in blood having increased activation, $\mathrm{V} \beta 21.3$ gene segment enrichment, and increased frequencies of activated cells that can interact with vasculature. It remains unclear whether these $\mathrm{T}$ cells reflect the phenotype of cells that did not exit into tissues and/or whether these activated $\mathrm{T}$ cells are directly related to the pathogenesis of disease. Further, while the Vbeta expansion and polyclonality data might suggest that no single classically recognized antigen is driving disease [16•], the true specificity of the activated $\mathrm{T}$ cells remains unknown.

In summary, MIS-C is manifested by increased cytokine production; increased PLA2G2A protein; decreases in total frequencies of monocytes, dendritic cells, and $\mathrm{T}$ cells; and increases in frequencies of activated $\mathrm{T}$ cells and plasmablasts.

\section{Immunopathogenesis}

The possibilities for immunopathogenesis of MIS-C remain broad (Fig. 1). The most striking and consistent feature identified by multiple groups has been expansion of $\mathrm{V} \beta 21.3+\mathrm{T}$ cells $[15 \bullet, 16 \bullet, 19 \bullet]$, although this finding is not present uniformly. The expansion is similar to that observed in superantigenic processes such as streptococcal toxic shock syndrome (STSS), and certainly, some clinical features of MIS-C overlap with STSS [16•]. Still, it remains unclear why such a syndrome would most prominently develop in the school-aged child-with relatively few cases in early childhood and in adulthood—given that a superantigen's ability to bind TCRVbeta segments to MHC alleles is not known to change with age [39]. Second, the 4-week time period between acute infection and disease is inconsistent with the antigen being from viral structures alone [40]. Still, SARS-CoV-2 RNA remains detectable in many patients at the time of hospitalization for MIS-C, and one study has suggested detectable antigen
Fig. 1 Potential immunopathogenesis of MIS-C. Created with Biorender.com

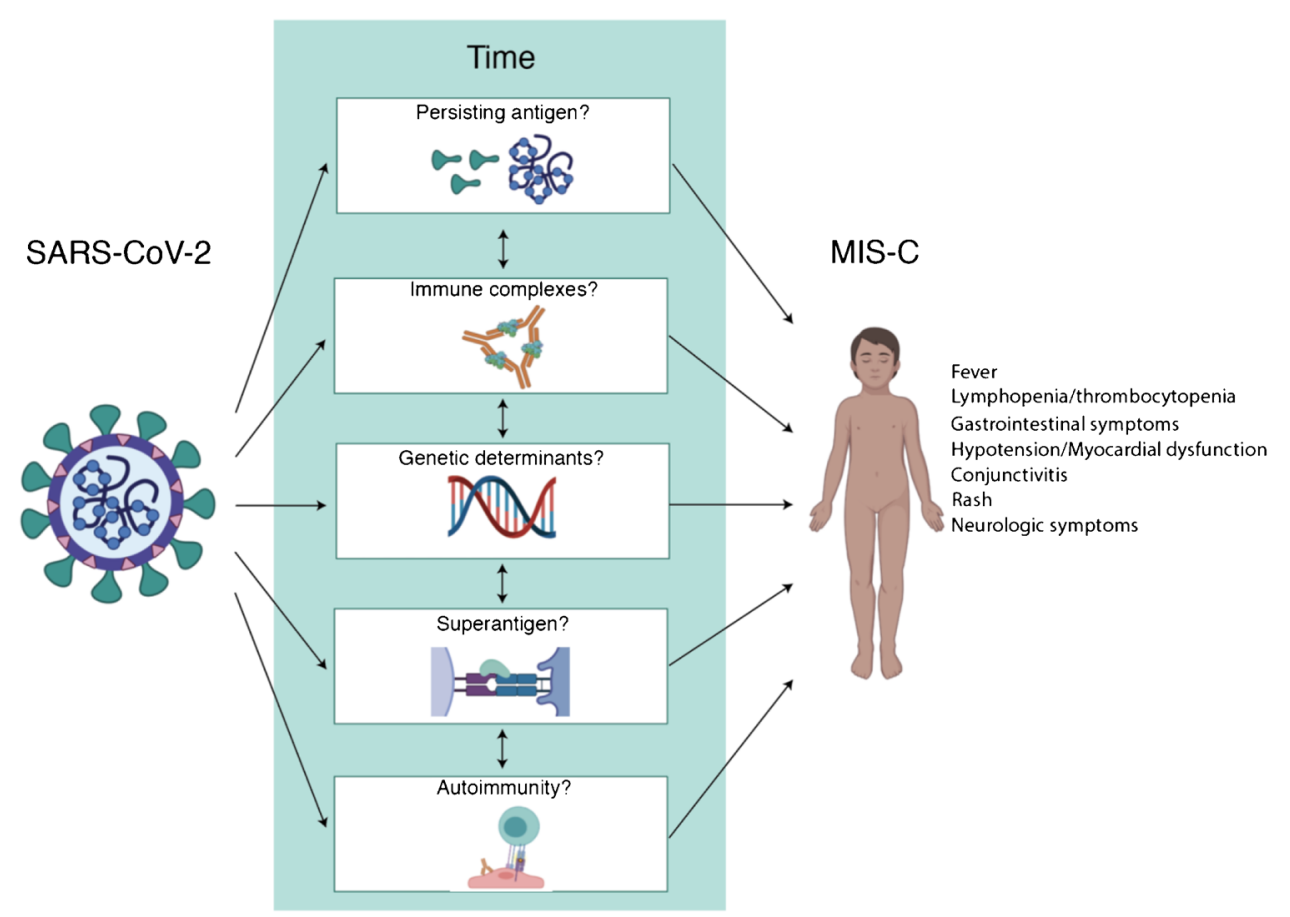


in circulation $[3 \bullet, 8 \bullet \bullet, 41]$. Differences in cellular tropism by patient age, viral variant, or other factors remain important avenues of investigation. Other possibilities include immune-activating structures or superantigens resulting from immune complexes formed with persisting

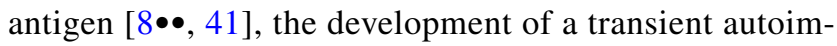
mune response $[12 \bullet \bullet, 14 \bullet \bullet, 15 \bullet$, or an otherwise dysregulated immune response that occurs around the time of viral clearance. Future work to evaluate each hypothesis will benefit from collaborative approaches to increase the diversity and sample size of patients as well as the adequacy of control populations.

\section{MIS-C and KD}

$\mathrm{KD}$ is a febrile illness of young childhood that can lead to infiltration of inflammatory cells into medium-sized arteries, particularly the coronary arteries, with a risk of myocardial infarction and sudden death. Because the etiology of KD has remained unknown for the past 50 years, the diagnosis has been based upon compatible clinical and laboratory features, and there is a broad differential diagnosis [42]. A subset of children with KD does not have all the clinical features identified in classic KD (so-called incomplete KD) and yet develops severe coronary artery disease, further complicating diagnosis. The existence of incomplete KD led the American Heart Association's Committee on Endocarditis, Rheumatic Fever, and Kawasaki Disease to propose a treatment algorithm for possible incomplete KD [42]. The purpose of the algorithm is to define clinical, laboratory, and echocardiographic features that support a possible diagnosis of incomplete KD when no other diagnosis can be established and to suggest when the benefit of treating for KD to prevent coronary artery aneurysms that could lead to lifelong heart disease likely outweighs the possible risk. It is not intended to provide definite diagnosis of KD, and the diagnosis cannot be considered confirmed in incomplete cases unless coronary artery inflammation with subsequent aneurysm formation develops.

When MIS-C cases were first identified, children with the illness were noted to have some of the clinical features of $\mathrm{KD}$, and some had mild coronary artery dilation in the acute febrile phase of illness. This led to initial speculation that MIS-C was somehow related to KD [43, 44]. However, the epidemiology of the two conditions is markedly different. Table 2 provides distinguishing features of acute COVID19, MIS-C, and KD. The presence of leukopenia, thrombocytopenia, hypotension, myocardial dysfunction, prominent gastrointestinal symptoms, and presentation at an older age favor MIS-C over KD as the diagnosis [6, 45]. The majority of children with KD are less than 5 years of age, and children of East Asian ancestry have the highest incidence [42]. In nations with the highest prevalence of KD, such as Japan, China, and Korea, MIS-C cases have been notably absent, and KD prevalence in the USA does not pattern MIS-C prevalence $[1 \bullet, 46,47]$. Perhaps the most compelling epidemiologic data indicating a lack of association between MIS-C and KD are the worldwide decrease in KD cases during COVID-19 outbreaks and mitigation strategies [1 $\bullet, 47$, $48,49 \bullet \bullet, 50,51]$. Masking and social distancing, which have reduced the prevalence of many respiratory viruses, presumably have decreased the prevalence of transmission of the as-yet-unidentified KD respiratory etiologic agent(s) [52].

Children with MIS-C rarely meet classic diagnostic criteria for $\mathrm{KD}$, typically only displaying the KD criteria of conjunctival injection and rash, features observed in many infectious and inflammatory illnesses of childhood. Moreover, the coronary artery dilation occurring in MIS-C is generally mild and is apparent during the initial febrile illness, rapidly resolving on short-term follow-up in most cases [53••, $54 \bullet \bullet$, similar to that observed in systemic onset juvenile idiopathic arthritis and rarely in children with other infections $[55,56]$. This is markedly different from the pattern of coronary artery dilation in $\mathrm{KD}$, which generally peaks after resolution of the febrile illness, during a time that the child appears clinically well [42]. This peak of coronary artery dilation in KD has been reported to occur at a median of 35 days after fever onset [57]. The difference in timing and severity of coronary artery dilation in the two conditions is likely related to different pathologic mechanisms. In MIS$\mathrm{C}$, coronary dilation may be due to increases in circulating cytokines with endothelial cell dysfunction and likely edema with mild dilation of the coronary arteries, while in KD, there is infiltration of the coronary arteries by inflammatory cells, leading to disruption of collagen and elastin fibers, and loss of structural integrity resulting in aneurysms of the arteries [58]. In the very few pathologic studies of the heart in cases of MIS-C or MIS-A published to date, inflammatory cells have been observed in interstitial spaces of the myocardium, without apparent infiltration into the coronary arteries $[59,60]$. Fortunately, myocardial function also appears to normalize in almost all children with MIS-C at short-term follow-up [53••]. Because of the difficulties in differentiating MIS-C from KD in individual cases, some patients reported to have had MIS-C likely had KD, and vice versa. Some studies examining the differences between pathogenesis of KD and MIS-C include children with incomplete KD in their studies, many of whom may have had non-KD illnesses. Given these caveats in accurate identification of both MIS-C and KD, it is difficult to know whether studies comparing immune dysregulation in MIS-C to KD are providing accurate data.

In studies performed in the pre-pandemic era, both innate and adaptive arms of the immune system were shown to 


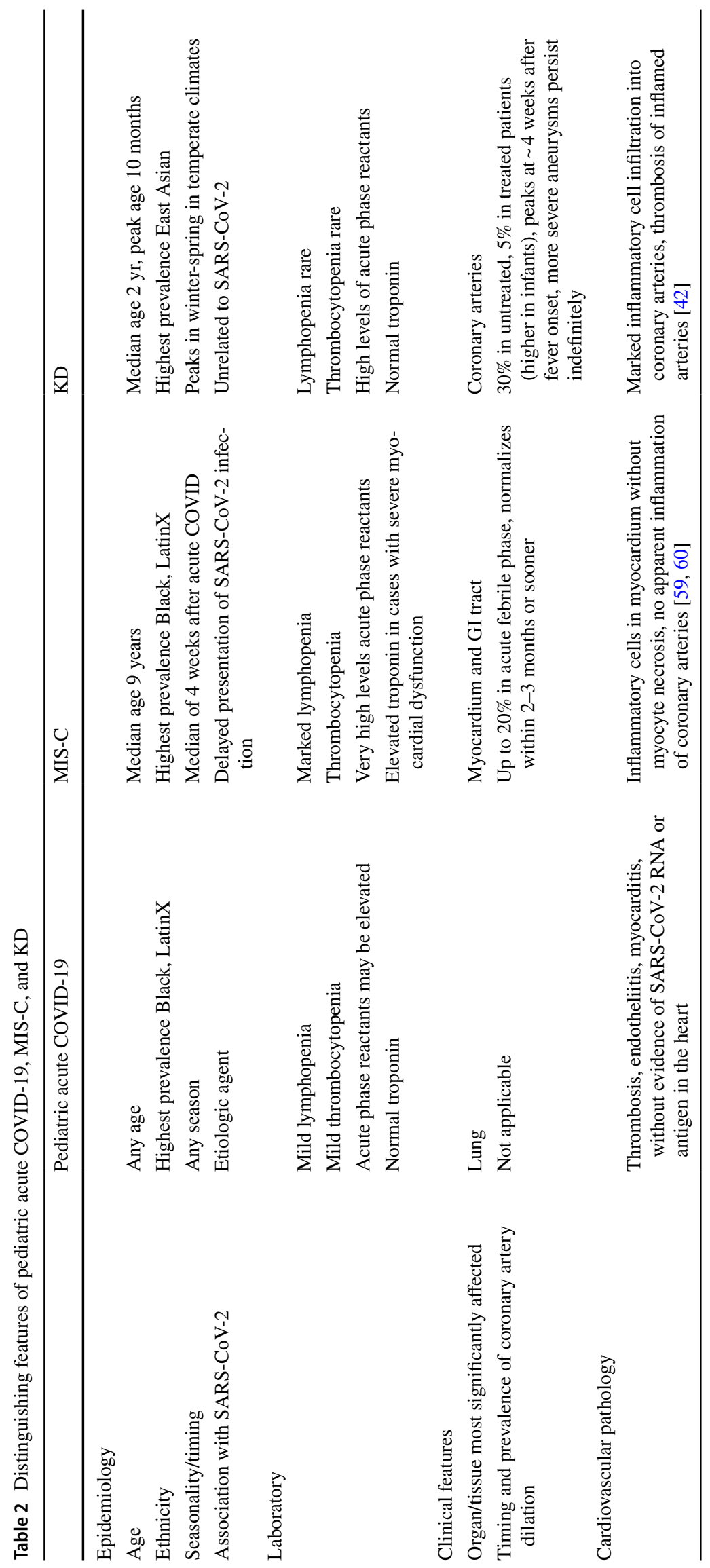


be activated in acute $\mathrm{KD}$, and the response is more typical of a conventional infectious agent than a superantigen [61]. In particular, early reports of $\mathrm{T}$ cell Vbeta skewing in acute KD peripheral blood that suggested a superantigen stimulus were not reproduced in many subsequent studies [62]. The lack of $\mathrm{T}$ cell Vbeta skewing in KD was recently reported to be a differentiating feature of KD compared with MIS-C [16•]. One study comparing plasma cytokine profiles in KD to MIS-C suggested a group of proteins differentiating KD, MIS-C, and acute COVID-19 infection [12••]. In this analysis, KD was associated with relative upregulation of CXCL9 and OSM and downregulation of ITGB6. In a separate, pre-pandemic study, CXCL9 was also observed as the most upregulated transcript in inflamed KD coronary arteries compared with childhood control coronary arteries, with OSM upregulation and ITGB6 downregulation also

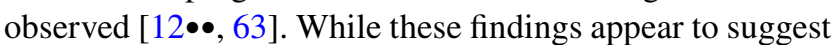
consistent cytokine features across $\mathrm{KD}$, one inherent difficulty in direct comparisons of cytokine levels between KD and MIS-C is the significantly different age groups affected by the disorders. For example, IL-17A was reported to be higher in KD compared with MIS-C [12••]; however, another report clearly demonstrated an inverse relationship between IL-17A levels and patient age [64], and KD children are significantly younger than MIS-C patients.

Over time, newer studies are clarifying the clinical and laboratory features that differentiate the two conditions, which should allow for improved pathogenesis studies in the future.

\section{Treatment}

The optimal treatment for MIS-C is unknown. Because of the clinical similarities between KD and MIS-C, treatment was modeled after that used in $\mathrm{KD}$, and intravenous gammaglobulin (IVIG) is often administered. Over time, anecdotal experience suggested that patients who received corticosteroid therapy with IVIG experienced more rapid improvement than those who did not. Several retrospective reviews support the clinical impression that myocardial function normalizes more quickly in children who receive corticosteroid with IVIG $[65,66,67 \bullet]$. A single study suggesting a lack of benefit of corticosteroids in MIS-C included a subset of patients who did not meet a standard MIS-C case definition and were negative for SARS-CoV-2 antibody, making a diagnosis of SARS-CoV-2-related illness unlikely in these patients and complicating interpretation of the results [68]. The optimal dose of corticosteroid for MIS-C remains unknown, but in two studies, doses in the $1-2-\mathrm{mg} / \mathrm{kg} / \mathrm{day}$ range were used $[65,66]$; many centers use a tapering dose regimen over a 2-3-week period. The role of IVIG in treatment remains unclear. Some centers have had success with corticosteroid treatment without IVIG in some patients [45] [69]. Patients who do not appear to respond to IVIG and corticosteroid are often treated with anakinra or infliximab, but the efficacy and role of these agents are also presently unknown. It also should be recognized that milder cases of MIS-C may be self-limited and could improve with supportive care alone [21].

\section{Conclusions}

MIS-C is a new pediatric illness that is a late complication of SARS-CoV-2 infection. KD should be considered in the differential diagnosis of MIS-C but is a separate disorder. The pathogenesis of MIS-C remains incompletely understood, but advances have been made in understanding the immune system components affected by the disorder and the immunomodulatory therapies that may hasten recovery. Continued work to explore potential immunopathologic mechanisms will be needed, with the most pressing question being whether the response is driven by a persisting component of the virus, a modified component of the virus, or whether the process is independent of persisting viral antigens and is, instead, virus-triggered autoimmunity. Most children recover completely, but long-term outcome studies are ongoing. It is hoped that universal vaccination of children against SARSCoV-2 will prevent pediatric cases of both COVID-19 and MIS-C in the future.

\section{References}

Papers of particular interest, published recently, have been highlighted as:

- Of importance

$\bullet$ Of major importance

1. Bailey LC, Razzaghi H, Burrows EK, et al (2021) Assessment of 135794 pediatric patients tested for severe acute respiratory syndrome coronavirus 2 across the United States. JAMA Pediatr 175:176-184. Reports a lack of association between MIS-C and KD diagnoses.

2. $\bullet$ Whittaker E, Bamford A, Kenny J, et al (2020) Clinical characteristics of 58 children with a pediatric inflammatory multisystem syndrome temporally associated with SARS-CoV-2. JAMA. https://doi.org/10.1001/jama.2020.10369. Describes clinical features of children in Europe with MIS-C early in the pandemic.

3. Godfred-Cato S, Bryant B, Leung J, et al (2020) COVID19-associated multisystem inflammatory syndrome in children - United States, March-July 2020. MMWR Morb Mortal Wkly Rep 69:1074-1080. Analyzes clinical differences between acute COVID-19 and MIS-C.

4. Feldstein LR, Rose EB, Horwitz SM, et al (2020) Multisystem inflammatory syndrome in U.S. children and adolescents. N Engl 
J Med. https://doi.org/10.1056/NEJMoa2021680. Reports clinical features of children in the USA with MIS-C.

5. Campbell JI, Roberts JE, Dubois M, Naureckas Li C, Sandora TJ, Lamb GS (2021) Non-SARS-CoV-2 infections among patients evaluated for MIS-C associated with COVID-19. Pediatr Infect Dis J 40:e90-e93. Demonstrates the wide differential diagnosis of MIS-C.

6. Roberts JE, Campbell JI, Gauvreau K, Lamb GS, Newburger J, Son MB, Dionne A (2021) Differentiating multisystem inflammatory syndrome in children: a single-centre retrospective cohort study. Arch Dis Child. https://doi.org/10.1136/archdischi 1d-2021-322290

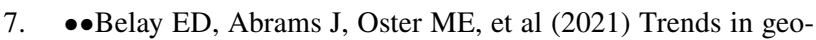
graphic and temporal distribution of US children with multisystem inflammatory syndrome during the COVID-19 pandemic. JAMA Pediatr. https://doi.org/10.1001/jamapediatrics.2021. 0630. Describes the epidemiology of MIS-C in the USA and its relationship to COVID-19 outbreaks.

8. $\bullet V$ Vella LA, Giles JR, Baxter AE, et al (2021) Deep immune profiling of MIS-C demonstrates marked but transient immune activation compared to adult and pediatric COVID-19. Sci Immunol. https://doi.org/10.1126/sciimmunol.abf7570. Provides a comprehensive analysis of immune dysregulation in MIS-C with adult and pediatric COVID-19 comparisons.

9. Payne AB, Gilani Z, Godfred-Cato S, et al. Incidence of multisystem inflammatory syndrome in children among US persons infected with SARS-CoV-2. JAMA Netw Open. 2021;4:e2116420.

10. Morris SB, Schwartz NG, Patel P, et al. Case series of multisystem inflammatory syndrome in adults associated with SARSCoV-2 infection - United Kingdom and United States, MarchAugust 2020. MMWR Morb Mortal Wkly Rep. 2020;69:1450-6.

11. Davogustto GE, Clark DE, Hardison E, Yanis AH, Lowery BD, Halasa NB, Wells QS. Characteristics associated with multisystem inflammatory syndrome among adults with SARS-CoV-2 infection. JAMA Netw Open. 2021;4:e2110323.

12•• Consiglio C, Cotugno N (2020) The immunology of multisystem inflammatory syndrome in children with COVID-19. Cell 183(4) 968-981. Reports immunologic features of MIS-C and potential differences with those of KD.

13•• Carter MJ, Fish M, Jennings A, et al (2020) Peripheral immunophenotypes in children with multisystem inflammatory syndrome associated with SARS-CoV-2 infection. Nat Med 26:1701-1707. Robust longitudinal immunophenotyping in MIS-C.

14.• Gruber CN, Patel RS, Trachtman R, et al (2020) Mapping systemic inflammation and antibody responses in multisystem inflammatory syndrome in children (MIS-C). Cell 183:982-995. e14. High-dimensional immunophenotyping in MIS-C.

15. Ramaswamy A, Brodsky NN, Sumida TS, et al (2021) Immune dysregulation and autoreactivity correlate with disease severity in SARS-CoV-2-associated multisystem inflammatory syndrome in children. Immunity 54:1083-1095.e7. Single-cell immunophenotyping of MIS-C and evidence of Vbeta skewing.

16. Moreews M, Le Gouge K, Khaldi-Plassart S, et al (2021) Polyclonal expansion of TCR Vbeta 21.3 CD4 and CD8 T cells is a hallmark of multisystem inflammatory syndrome in children. Sci Immunol. https://doi.org/10.1126/sciimmunol.abh1516. Identifies and phenotypes cells with skewing of $T$ cell receptor usage in MIS-C with COVID-19 and Kawasaki disease controls.

17. Diorio C, Henrickson SE, Vella LA, et al (2020) Multisystem inflammatory syndrome in children and COVID-19 are distinct presentations of SARS-CoV-2. J Clin Invest 130:5967-5975. Describes immunologic features differentiating acute COVID-19 from MIS-C.
18. •Pierce CA, Preston-Hurlburt P, Dai Y, et al (2020) Immune responses to SARS-CoV-2 infection in hospitalized pediatric and adult patients. Sci Transl Med. https://doi.org/10.1126/ scitranslmed.abd5487. Robust serologic data with comparisons to adult and pediatric COVID-19.

19. •Porritt RA, Paschold L, Rivas MN, et al (2021) HLA class I-associated expansion of TRBV11-2 T cells in multisystem inflammatory syndrome in children. J Clin Invest. https://doi. org/10.1172/JCI146614. Demonstrates Vbeta skewing in MIS-C with febrile controls.

20. Diorio C, Shraim R, Vella LA, et al (2021) Proteomic profiling of MIS-C patients reveals heterogeneity relating to interferon gamma dysregulation and vascular endothelial dysfunction. medRxiv. https://doi.org/10.1101/2021.04.13.21255439. Broad proteomic profiling in MIS-C vs COVID-19 that identifies elevation of PLA2G2A in MIS-C.

21. Lee PY, Day-Lewis M, Henderson LA, et al. Distinct clinical and immunological features of SARS-CoV-2-induced multisystem inflammatory syndrome in children. J Clin Invest. 2020;130:5942-50.

22. Rodriguez-Smith JJ, Verweyen EL, Clay GM, et al (2021) Inflammatory biomarkers in COVID-19-associated multisystem inflammatory syndrome in children, Kawasaki disease, and macrophage activation syndrome: a cohort study. The Lancet Rheumatology. https://doi.org/10.1016/S2665-9913(21) 00139-9

23. Kuypers FA, Rostad CA, Anderson EJ, et al (2021) Secretory phospholipase A2 in SARS-CoV-2 infection and multisystem inflammatory syndrome in children (MIS-C). Exp Biol Med 15353702211028560

24. Cheung EW, Zachariah P, Gorelik M, Boneparth A, Kernie SG, Orange JS, Milner JD. Multisystem inflammatory syndrome related to COVID-19 in previously healthy children and adolescents in New York City. JAMA. 2020;324:294-6.

25. Lee PY, Platt CD, Weeks $S$, et al. Immune dysregulation and multisystem inflammatory syndrome in children (MIS-C) in individuals with haploinsufficiency of SOCS1. J Allergy Clin Immunol. 2020;146:1194-1200.e1.

26. Chou J, Platt CD, Habiballah S, et al (2021) Mechanisms underlying genetic susceptibility to multisystem inflammatory syndrome in children (MIS-C). J Allergy Clin Immunol. https://doi. org/10.1016/j.jaci.2021.06.024

27. Pérez-Gómez A, Vitallé J, Gasca-Capote C, et al (2021) Dendritic cell deficiencies persist seven months after SARSCoV-2 infection. Cell Mol Immunol. https://doi.org/10.1038/ s41423-021-00728-2

28. Hall MW. Immune modulation in pediatric sepsis. Pediatr Crit Care Med. 2019;8:42-50.

29. Meisel C, Schefold JC, Pschowski R, et al. Granulocyte-macrophage colony-stimulating factor to reverse sepsis-associated immunosuppression: a double-blind, randomized, placebocontrolled multicenter trial. Am J Respir Crit Care Med. 2009;180:640-8.

30. Anderson EM, Diorio C, Goodwin EC, et al (2020) SARSCoV-2 antibody responses in children with MIS-C and mild and severe COVID-19. J Pediatric Infect Dis Soc. https://doi.org/10. 1093/jpids/piaa161

31. Rostad CA, Chahroudi A, Mantus G, et al (2020) Quantitative SARS-CoV-2 serology in children with multisystem inflammatory syndrome (MIS-C). Pediatrics. https://doi.org/10.1542/peds. 2020-018242

32. Bartsch YC, Wang C, Zohar T, et al. Humoral signatures of protective and pathological SARS-CoV-2 infection in children. Nat Med. 2021;27:454-62. 
33. McElroy AK, Akondy RS, Davis CW, et al. Human Ebola virus infection results in substantial immune activation. Proc Natl Acad Sci U S A. 2015;112:4719-24.

34. Wrammert J, Onlamoon N, Akondy RS, et al. Rapid and massive virus-specific plasmablast responses during acute dengue virus infection in humans. J Virol. 2012;86:2911-8.

35. Wec AZ, Haslwanter D, Abdiche YN, et al. Longitudinal dynamics of the human B cell response to the yellow fever 17D vaccine. Proc Natl Acad Sci U S A. 2020;117:6675-85.

36. Nash MC, Shah V, Reader JA, Dillon MJ. Anti-neutrophil cytoplasmic antibodies and anti-endothelial cell antibodies are not increased in Kawasaki disease. Br J Rheumatol. 1995;34:882-7.

37. Guzman J, Fung M, Petty RE. Diagnostic value of anti-neutrophil cytoplasmic and anti-endothelial cell antibodies in early Kawasaki disease. J Pediatr. 1994;124:917-20.

38. Weisberg SP, Connors TJ, Zhu Y, et al. Distinct antibody responses to SARS-CoV-2 in children and adults across the COVID-19 clinical spectrum. Nat Immunol. 2021;22:25-31.

39. Sharma H, Smith D, Turner CE, Game L, Pichon B, Hope R, Hill R, Kearns A, Sriskandan S (2018) Clinical and molecular epidemiology of staphylococcal toxic shock syndrome in the United Kingdom. Emerg Infect Dis. https://doi.org/10.3201/ eid2402.170606

40. Cheng MH, Zhang S, Porritt RA, Noval Rivas M, Paschold L, Willscher E, Binder M, Arditi M, Bahar I. Superantigenic character of an insert unique to SARS-CoV-2 spike supported by skewed TCR repertoire in patients with hyperinflammation. Proc Natl Acad Sci U S A. 2020;117:25254-62.

41. Yonker LM, Gilboa T, Ogata AF, et al (2021) Multisystem inflammatory syndrome in children is driven by zonulin-dependent loss of gut mucosal barrier. J Clin Invest. https://doi.org/10. 1172/JCI149633

42. McCrindle BW, Rowley AH, Newburger JW, et al. Diagnosis, treatment, and long-term management of Kawasaki disease: a scientific statement for health professionals from the American Heart Association. Circulation. 2017;135:e927-99.

43. Verdoni L, Mazza A, Gervasoni A, Martelli L, Ruggeri M, Ciuffreda M, Bonanomi E, D’Antiga L (2020) An outbreak of severe Kawasaki-like disease at the Italian epicentre of the SARSCoV-2 epidemic: an observational cohort study. Lancet. https:// doi.org/10.1016/S0140-6736(20)31103-X

44. Ouldali N, Pouletty M, Mariani P, et al (2020) Emergence of Kawasaki disease related to SARS-CoV-2 infection in an epicentre of the French COVID-19 epidemic: a time-series analysis. Lancet Child Adolesc Health. https://doi.org/10.1016/S23524642(20)30175-9

45. Bar-Meir M, Guri A, Godfrey ME, Shack AR, Hashkes PJ, Goldzweig O, Megged O. Characterizing the differences between multisystem inflammatory syndrome in children and Kawasaki disease. Sci Rep. 2021;11:13840.

46. Kim Y-J, Park H, Choi YY, Kim YK, Yoon Y, Kim K-R, Choi EH (2020) Defining association between COVID-19 and the multisystem inflammatory syndrome in children through the pandemic. Journal of Korean Medical Science. https://doi.org/ 10.3346/jkms.2020.35.e204

47. Iio K, Uda K, Hataya H, Yasui F, Honda T, Sanada T, Yamaji K, Kohara M, Itokawa M, Miura M. Kawasaki disease or Kawasakilike disease: influence of SARS-CoV-2 infections in Japan. Acta Paediatr. 2021;110:600-1.

48. Shulman S, Geevarghese B, Kim KY, Rowley A (2021) The impact of social distancing for COVID-19 upon diagnosis of Kawasaki disease. J Pediatric Infect Dis Soc. https://doi.org/10. 1093/jpids/piab013

49. $\bullet$ Kang JM, Kim YE, Huh K, Hong J, Kim DW, Kim MY, Jung SY, Kim JH, Jung J, Ahn JG (2021) Reduction in Kawasaki disease after nonpharmaceutical interventions in the
COVID-19 era: a nationwide observational study in Korea. Circulation. https://doi.org/10.1161/CIRCULATIONAHA. 121.054785. Reports the decrease in KD diagnoses across Korea during COVID mitigation strategies.

50. Iio K, Matsubara K, Miyakoshi C, Ota K, Yamaoka R, Eguchi J, Matsumura O, Okutani T, Ueda I, Nishiyama M. Incidence of Kawasaki disease before and during the COVID-19 pandemic: a retrospective cohort study in Japan. BMJ Paediatr Open. 2021;5:e01034.

51. Ae R, Shibata Y, Kosami K, Nakamura Y, Hamada H (2021) Kawasaki disease and pediatric infectious diseases during the coronavirus disease 2019 pandemic. J Pediatr. https://doi.org/ 10.1016/j.jpeds.2021.07.053

52. Rowley AH, Baker SC, Arrollo D, et al. A protein epitope targeted by the antibody response to Kawasaki disease. J Infect Dis. 2020;222:158-68.

$53 \bullet$ Farooqi KM, Chan A, Weller RJ, et al (2021) Longitudinal outcomes for multisystem inflammatory syndrome in children. Pediatrics. https://doi.org/10.1542/peds.2021-051155. Describes generally excellent outcomes for children with MIS-C.

54.•• Feldstein LR, Tenforde MW, Friedman KG, et al (2021) Characteristics and outcomes of US children and adolescents with multisystem inflammatory syndrome in children (MIS-C) compared with severe acute COVID-19. JAMA 325:1074-1087. Describes generally excellent outcomes for children with MIS-C, including the lack of giant aneurysms.

55. Binstadt BA, Levine JC, Nigrovic PA, Gauvreau K, Dedeoglu F, Fuhlbrigge RC, Weindling SN, Newburger JW, Sundel RP. Coronary artery dilation among patients presenting with systemiconset juvenile idiopathic arthritis. Pediatrics. 2005;116:e89-93.

56. Muniz JC, Dummer K, Gauvreau K, Colan SD, Fulton DR, Newburger JW. Coronary artery dimensions in febrile children without Kawasaki disease. Circ Cardiovasc Imaging. 2013;6:239-44.

57. Tsuda E, Hashimoto S. Time course of coronary artery aneurysms in Kawasaki disease. J Pediatr. 2021;230:133-139.e2.

58. Orenstein JM, Shulman ST, Fox LM, et al. Three linked vasculopathic processes characterize Kawasaki disease: a light and transmission electron microscopic study. PLoS One. 2012;7:e38998.

59. Duarte-Neto AN, Caldini EG, Gomes-Gouvêa MS, et al. An autopsy study of the spectrum of severe COVID-19 in children: from SARS to different phenotypes of MIS-C. EClinicalMedicine. 2021;35:100850.

60. Fox SE, Lameira FS, Rinker EB, Vander Heide RS. Cardiac endotheliitis and multisystem inflammatory syndrome after COVID-19. Ann Intern Med. 2020;173:1025-7.

61. Rowley AH, Shulman ST. The epidemiology and pathogenesis of Kawasaki disease. Front Pediatr. 2018;6:374.

62. Rowley AH, Shulman ST. New developments in the search for the etiologic agent of Kawasaki disease. Curr Opin Pediatr. 2007;19:71-4.

63. Rowley AH, Wylie KM, Kim KY, et al. The transcriptional profile of coronary arteritis in Kawasaki disease. BMC Genomics. 2015;16:1076.

64. Pierce CA, Sy S, Galen B, Goldstein DY, Orner E, Keller MJ, Herold KC, Herold BC (2021) Natural mucosal barriers and COVID-19 in children. JCI Insight. https://doi.org/10.1172/jci. insight.148694

65. Belhadjer Z, Auriau J, Méot M, Oualha M, Renolleau S, Houyel $\mathrm{L}$, Bonnet D. Addition of corticosteroids to immunoglobulins is associated with recovery of cardiac function in multi-inflammatory syndrome in children. Circulation. 2020;142:2282-4.

66. Ouldali N, Toubiana J, Antona D, et al. Association of intravenous immunoglobulins plus methylprednisolone vs 
immunoglobulins alone with course of fever in multisystem inflammatory syndrome in children. JAMA. 2021;325:855-64.

67 Son MBF, Murray N, Friedman K, et al (2021) Multisystem inflammatory syndrome in children - initial therapy and outcomes. N Engl J Med 385:23-34. The above 3 studies report more rapid improvement in children with MIS-C treated with corticosteroid therapy.

68. McArdle AJ, Vito O, Patel H, et al. Treatment of multisystem inflammatory syndrome in children. N Engl J Med. 2021;385:11-22.

69. V Vukomanovic S Krasic S Prijic S Ninic S Popovic G Petrovic S Ristic R Simic I CerovicD Nesic 2021 Recent experience: corticosteroids as a first-line therapy in children with multisystem inflammatory syndrome and COVID-19-related myocardial damage Pediatr Infect Dis J https://doi.org/10.1097/INF.00000 00000003260

Publisher's Note Springer Nature remains neutral with regard to jurisdictional claims in published maps and institutional affiliations. 